\title{
Manifestação do Duplo Padrão Sexual nas sociedades ocidentais (2011-2017): uma revisão abrangente
}

Manifestation of the Sexual Double Standard in Western Societies (2011-2017): A Scoping Review

Manifestation du double standard sexuel dans les sociétés occidentales

(2011-2017) : un examen de la portée

Helena Delgado Amaro, Maria-João Alvarez e Joaquim Armando Ferreira

(2) OpenEdition

Journals

Edição electrónica

URL: https://journals.openedition.org/rccs/11509

DOI: $10.4000 /$ rccs. 11509

ISSN: 2182-7435

\section{Editora}

Centro de Estudos Sociais da Universidade de Coimbra

Edição impressa

Data de publição: 1 maio 2021

Paginação: 53-78

ISSN: 0254-1106

Refêrencia eletrónica

Helena Delgado Amaro, Maria-João Alvarez e Joaquim Armando Ferreira, «Manifestação do Duplo Padrão Sexual nas sociedades ocidentais (2011-2017): uma revisão abrangente», Revista Crítica de Ciências Sociais [Online], 124 | 2021, publicado a 26 maio 2021, consultado a 28 maio 2021. URL: http://journals.openedition.org/rccs/11509; DOl: https://doi.org/10.4000/rccs.11509 


\section{HELENA DELGADO AMARO, MARIA-JOÃO ALVAREZ, JOAQUIM ARMANDO FERREIRA}

\section{Manifestação do Duplo Padrão Sexual nas sociedades ocidentais (2011-2017): uma revisão abrangente}

Esta revisão abrangente analisa investigação publicada entre 2011 e $2017(n=46)$, que averiguou a emergência e a variação do Duplo Padrão Sexual (DPS) em sociedades ocidentais. Observa-se uma consistente manifestação do DPS na avaliação de condutas sexuais diversas. Há igualmente evidência de padrões sexuais alternativos positivos (igualitários liberais) e negativos (igualitários conservadores e invertidos). A emergência de DPS e a variação com padrões negativos indica que, nas sociedades ocidentais, a experiência (hetero)sexual é marcada por desigualdade de género e por padrões sexuais restritivos e punitivos. E, porque estes padrões podem limitar a liberdade sexual e o acesso de homens e mulheres a uma sexualidade positiva, devem ser alvo de desconstrução e substituição por outros, equitativos.

Palavras-chave: conduta sexual; duplo padrão sexual; género; sexualidade.

\section{Introdução}

A noção de Duplo Padrão Sexual (DPS) foi introduzida por Ira Reiss para descrever a tendência a sancionar mulheres, mas não homens, pelo envolvimento em sexo pré-marital. Hoje continua a definir um padrão diferencial de avaliação da conduta sexual, mais permissivo face à sexualidade masculina e mais restritivo face à feminina (Reiss, 1960). Há provas da sua atenuação para alguns comportamentos (e.g., frequência da atividade sexual, sexo oral e anal) e atitudes sexuais (e.g., sexo extradiádico, masturbação), mas igualmente da sua contínua manifestação para uma multiplicidade de comportamentos, como por exemplo o sexo ocasional - sem compromisso e limitado no tempo (e.g., uma noite) - ou o elevado número de parceiros sexuais (Bordini e Sperb, 2013; Crawford e Popp, 2003; Petersen e Hyde, 2010, 2011).

ODPS pode ser entendido como uma expressão do género - crença socialmente partilhada de que homens e mulheres são diferentes - na sexualidade. 
É informado e, em certa medida, legitimado pelos estereótipos e papéis sexuais de género, os quais definem as características e os comportamentos sexuais apropriados e aceites/proibidos para homens e mulheres e podem ser resumidos nas dicotomias homem sexual vs. mulher emocional e homem ativo vs. mulher passiva, respetivamente (Blakemore et al., 2008; Howard e Hollander, 1997). Alicerçado nestas construções, o DPS ensina aos homens que a sexualidade é a chave da masculinidade e às mulheres que a sexualidade deve ser minimizada e controlada (McCarthy e Bodnar, 2005). Contudo, o desvio pode pôr em causa feminilidade, masculinidade e reputação sexual de ambos e o DPS, ao mesmo tempo que reforça a desigualdade de género, pode condicionar a liberdade sexual de homens e mulheres, o que revela, a par da sua manifestação ao longo dos anos, a importância de conhecer - e combater - este padrão (Bordini e Sperb, 2013; Crawford e Popp, 2003; Fasula et al., 2014). Compreender e agir na desconstrução do DPS é tanto mais importante quanto, por um lado, apesar da atenuação da aceitação pessoal do padrão (DPS pessoal) - a mais frequentemente explorada -, a sua existência social (DPS social) é reconhecida, e, por outro, os padrões sexuais alternativos que têm emergido são, em alguns casos, positivos mas, noutros, negativos (Bordini e Sperb, 2013; Crawford e Popp, 2003). São padrões alternativos o Padrão Sexual Singular (PSS) que prescreve igual liberdade sexual e julgamento/ /punição de homens e mulheres, e que pode ser ou mais liberal ou mais restritivo e punitivo (PSS liberal e PSS conservador), e o Duplo Padrão Sexual Invertido (DPS Invertido) que prescreve menor liberdade sexual, julgamento mais negativo e punição mais severa dos homens do que das mulheres.

Para uma atualização do conhecimento sobre DPS propõe-se, por isso, analisar trabalhos de pesquisa produzidos entre 2011 e 2017 em sociedades ocidentais e apresentar um resumo integrado dos principais resultados obtidos sobre a emergência do padrão e sobre a sua variação com padrões sexuais alternativos.

\section{Método usado na revisão abrangente}

São objetivos desta revisão abrangente examinar investigação sobre DPS seguindo as etapas definidas por Arksey e O'Malley (2005): 1) identificação da questão de investigação; 2) identificação de estudos relevantes; 3) seleção de estudos; 4) mapeamento dos dados; 5) resumo dos resultados.

A questão de investigação tomou como referência para a delimitação do período temporal e área geográfica as anteriores revisões de literatura 
sobre DPS (Bordini e Sperb, 2013; Crawford e Popp, 2003). Os autores interrogaram-se sobre o que revela a investigação publicada entre 1 de janeiro de 2011 e 30 de abril de 2017 sobre manifestação de DPS e variação com padrões sexuais alternativos, publicada e realizada nos Estados Unidos da América (EUA), Canadá, Europa Ocidental e Austrália.

Para a identificação dos estudos relevantes foi conduzida uma pesquisa na Biblioteca de Conhecimento Online (b-on), uma base de dados que disponibiliza conteúdos publicados por diferentes editores (e.g., Elsevier, Sage, Springer, Taylor \& Francis, Clarivate Analytics, Wiley), usando o termo de pesquisa "sexual double standard" nos campos título, resumo e termos do assunto, e o operador booleano "OR". Além do período temporal e da área geográfica, limitou-se a pesquisa à investigação escrita em língua inglesa, a par da área temática, do tipo de investigação (foi apenas incluída investigação empírica), das características dos estudos (e.g., objetivos, contextos/perspetivas analisadas) e da adequação aos objetivos da revisão abrangente (e.g., excluída operacionalização de DPS afastada da definição considerada, tratamento secundário da variável).

A pesquisa inicial resultou em 346 entradas, reduzidas para 120 depois de excluídas referências duplicadas e para 73 depois de excluídas: três por não conterem o termo de pesquisa ou não serem escritas em língua inglesa, 20 por não cumprirem o critério relativo à área geográfica, 12 por não cumprirem o critério tipo de investigação e outras 12 o critério área temática. Da análise detalhada dessas 73 referências excluíram-se oito (e.g., duplo padrão não sexual, medidas DPS, DPS secundário), tendo-se chegado a 65 artigos que cumpriram os critérios de elegibilidade (Diagrama 1). O processo de pesquisa e seleção foi inicialmente conduzido pela primeira autora deste artigo e depois revisto pela segunda, como garantia de rigor.

Da leitura integral e análise dos artigos selecionados, realizada pelos três autores, concluiu-se sobre dois tipos de resultados: os relativos à manifestação/variação do DPS $(n=46)$, cujos resultados são analisados neste artigo, e os relativos aos efeitos do DPS $(n=19)$, não considerados na presente revisão abrangente. ${ }^{1}$ Extraiu-se e compilou-se informação relevante (autor, data, área geográfica, objetivos, métodos e resultados), que foi organizada com base nos comportamentos/contextos/grupos em análise.

${ }^{1}$ Investigação portuguesa produzida entre 2011 e 2017 faria parte deste grupo. 


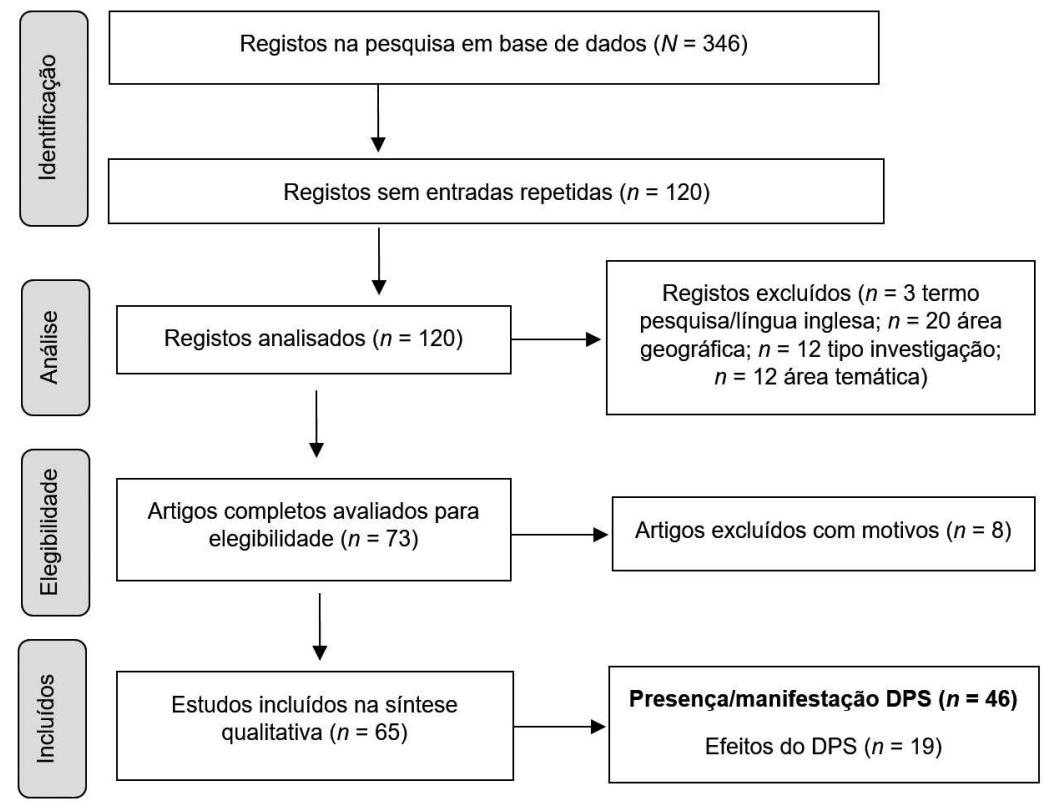

DIAGRAMA 1 - Adaptação das orientações PRISMA (Preferred Reporting Items for Systematic Reviews and Meta-Analyses), propostas por Moher et al. (2009), à decisão sobre os artigos a incluir na revisão abrangente

Fonte: Elaboração própria.

\section{Resultados sobre DPS e padrões sexuais alternativos}

Os resultados organizaram-se em dois grandes temas que dizem respeito, respetivamente, à evidência de DPS e padrões alternativos para diferentes comportamentos e grupos - Emergências e Variações Gerais do DPS - e para o caso particular do sexo ocasional entre estudantes universitários (e outros) - Emergências e Variações Específicas do DPS. A divisão justificou-se pelo elevado número de trabalhos que analisou a aceitabilidade da ocasionalidade em homens e mulheres e pelo facto de este ser um padrão relacional cada vez mais comum no contexto universitário (Garcia et al., 2012).

Os estudos exploraram sobretudo a aceitação pessoal do DPS e menos o reconhecimento da sua existência social e, por isso, os resultados correspondentes ao DPS pessoal ou a casos em que não se pôde distinguir entre dimensões foram identificados apenas como DPS, enquanto os resultados sobre DPS social foram especificamente identificados como tal. 


\subsection{Emergências e variações gerais do DPS}

A secção divide-se em: 1) investigação sobre emergência/variação do DPS conduzida com adolescentes e jovens (amostras entre os 11/12 anos até cerca de 25 anos de idade); 2) com jovens adultos e adultos (amostras a partir dos 18 anos de idade); e 3) investigação que relaciona emergência/variação do DPS com as características de quem avalia/é avaliado.

\subsubsection{Adolescentes e jovens}

A evidência de DPS foi reunida em dois estudos desenvolvidos nos EUA. No primeiro, McClintock (2011) concluiu que as mulheres, mais do que os homens, procuravam relacionamentos comprometidos/exclusivos, referiam menos parceiros sexuais e adiavam a atividade sexual dentro do relacionamento - posições a apontar para estereótipos e papéis sexuais de género e indiretamente para DPS. No segundo, Kreager et al. (2016) mostraram que a aceitação das raparigas pelos pares diminuía, e a dos rapazes aumentava, com o envolvimento em comportamento sexual "pesado" (relações sexuais), ao passo que o envolvimento em comportamento sexual "leve" (curtir) fazia aumentar a aceitação das raparigas, mas diminuir a dos rapazes entre pares masculinos. Os autores consideraram que a última observação era reveladora de DPS Invertido mas, em nossa opinião, ela deve ser tomada, como as anteriores, enquanto expressão de DPS tradicional dado que este padrão pressupõe que o envolvimento em relações sexuais é prova de masculinidade e que, a não acontecer, pode fazer diminuir o estatuto dos homens entre os pares.

Em dois outros trabalhos com amostras americanas foi reunida evidência da emergência de DPS e da sua variação com padrões sexuais alternativos. Young et al. (2016) observaram que a avaliação que adolescentes faziam de alvos femininos ou masculinos (Between Subject Design ou BSD), em cenários hipotéticos (Person Perception Task ou PPT) sem referência a sexo ou com referência a envolvimento romântico com/sem atividade sexual, era mais negativa para aqueles que optavam por manter relações sexuais (PSS conservador) e para os alvos femininos (DPS) no grupo das mulheres. Já Lyons et al. (2011) concluíram, em dois estudos com amostras femininas, sobre a presença de um PSS liberal a alternar com um DPS social e pessoal. Os investigadores observaram que as participantes com elevado número de parceiros sexuais não se sentiam diferentes dos seus pares em termos de popularidade, número de amigos e autoestima, mas que algumas reconheciam o DPS no contexto escolar alargado e assumiam adotá-lo na avaliação do comportamento desse grupo, mas não do seu ou do de amigas. 
Por fim, três estudos acrescentaram ainda informação sobre a possível influência da sociedade e cultura americanas na emergência do DPS, partindo do pressuposto de que elas são marcadas por algum conservadorismo sexual. De facto, o debate de adolescentes/jovens latino-americanos em torno de fotografias (photovoice) levou Gubrium e Torres (2013) a concluir sobre a presença do DPS para as questões da expressão do desejo/ /prazer, agência ou proteção sexuais, e a considerá-la potencial reflexo da influência das culturas latina e americana. Tong (2013) sugeriu uma direção contrária, indicando que a exposição de adolescentes/jovens de ascendência asiática à cultura americana conduzia à adoção de normas sexuais mais liberais (especialmente entre mulheres), e não de DPS (início da atividade sexual e múltiplos parceiros). Já Chmielewski et al. (2017) observaram que, em artigos noticiosos sobre sexualidade adolescente, a sexualidade feminina era associada a risco e responsabilização pela proteção e pelas consequências da atividade sexual. Os discursos mediáticos reconheciam a agência sexual feminina, mas reproduziam a ideia tradicional de que as mulheres devem ser capazes de controlar a sexualidade e o risco sexual.

No que respeita a investigação produzida em sociedades europeias com adolescentes e jovens, dois trabalhos de pesquisa apontaram para a presença do DPS no contexto da interação/comunicação digital. Ringrose et al. (2013) observaram-no na avaliação que adolescentes fizeram da troca de imagens sexuais (sexting). Para os rapazes, possuir, trocar, mostrar imagens de raparigas foi avaliado positivamente e considerado fonte de popularidade, enquanto para as raparigas, tirar, partilhar ou publicar imagens sexuais foi associado a risco/culpa/vergonha, descrevendo as mulheres que o fazem como skets (mulher muito promíscua, "suja", sem valor) que não se dão ao respeito. Do mesmo modo, os dois estudos de Mascheroni et al. (2015) apontaram para a manifestação de DPS na apresentação da identidade online (fotografias) e na interação de adolescentes em redes sociais, mas também sugeriram alguma relativização do padrão. Os investigadores concluíram que, apesar da erotização das fotografias ser comum aos dois sexos, os rapazes sancionavam as raparigas por posarem de forma sexy (e.g., indicador de "certo tipo de rapariga"), ao passo que algumas mulheres pareciam secundarizar o DPS e priorizar a aceitação dos pares (e.g., conformidade com padrões de beleza sexualizados). A importância dos pares nos discursos sobre sexualidade e normas sexuais foi revelada neste último resultado e vai ao encontro do constatado por Bongardt et al. (2017) com base na conversação de díades de amigos/amigas sobre sexualidade. O desvio (sexualidade de risco) - mais expressivo nas díades masculinas - e a normatividade (sexualidade saudável) - mais expressiva 
nas femininas - associaram-se a normas sexuais percebidas nos pares. Simultaneamente, esta diferença e a associação de desvio e normatividade a diferentes normas (nas díades masculinas) e apenas a aprovação/reprovação (nas femininas) podem ser tomadas como indicadores indiretos de DPS. Num outro sentido, mas igualmente a sublinhar a presença indireta de DPS nos discursos produzidos na Europa Ocidental está o trabalho de Martin et al. (2014). Os investigadores concluíram que, em artigos noticiosos sobre saúde sexual, os homens eram frequentemente descritos como mais promíscuos e propensos ao envolvimento em comportamento de risco, enquanto às mulheres era atribuída a responsabilidade pela saúde sexual, discursos a refletir estereótipos e papéis sexuais de género, na base do DPS. Por fim, três estudos reúnem evidência de DPS e padrões sexuais alternativos, sendo que um deles destaca a possibilidade de haver diferenças no modo como adolescentes e jovens experienciam a sexualidade em sociedades e culturas europeias particulares. Fjær et al. (2015) observaram que, quando consideravam relacionamentos ocasionais na celebração da conclusão do ensino secundário, os jovens evitavam criticar mulheres incapazes de autocontrolo (PSS liberal), mas admitiam que estas podiam ser estigmatizadas (DPS social). Esta variação entre padrões foi igualmente visível na combinação que as mulheres participantes faziam entre discursos de tolerância e distanciamento face a essas outras mulheres, e na afirmação dos participantes homens de maior liberdade face a prescrições da masculinidade, apesar do reconhecimento do DPS. Damme e Biltereyst (2013) também observaram a emergência de padrões alternativos a variar com o reconhecimento do DPS social, mas distinguiram ainda um DPS pessoal residual. Adolescentes e jovens mostraram-se tolerantes face ao sexo ocasional, mas distanciaram-se da prática; defenderam igualdade na troca repetida de parceiros, mas reconheceram ser menos tolerada e na base de rotulagem mais negativa de mulheres do que de homens; e alguns assumiram adotar o DPS na avaliação de conhecidos mais do que na de amigos. Já no trabalho de Franz et al. (2016), discursos sobre sexualidade - enfatizando responsabilidade, respeito e prazer partilhados - apresentaram-se como dominantes entre jovens alemães e uma alternativa entre ingleses, cujos discursos, mais frequentemente, revelavam negociação entre o ideal relacional e a realidade ocasional do sexo. Não obstante, a igual valorização da partilha e da relação, em ambos os casos, aponta para posições mais igualitárias, sendo a alternância com o DPS pouco evidente.

Entre adolescentes e jovens americanos e europeus, o DPS emerge, portanto, de forma consistente e alterna, em alguns casos, com um PSS conservador e um PSS liberal (Tabela 1). 
60| Helena Delgado Amaro, Maria-João Alvarez, Joaquim Armando Ferreira

TABELA 1 - Emergência e variação do DPS, entre adolescentes e jovens, e jovens adultos e adultos, para diferentes condutas sexuais

\begin{tabular}{|c|c|c|c|c|}
\hline & Autoria da pesquisa & Tipo de estudo/método & População/amostra & Resultados \\
\hline \multirow{14}{*}{ 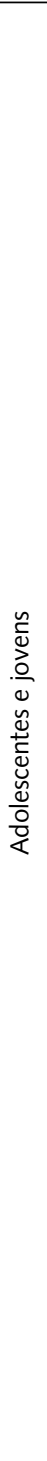 } & McClintock (2011) & $\begin{array}{l}\text { Misto - análise } \\
\text { quantitativa } \\
\text { entrevistas }\end{array}$ & $\begin{array}{l}\text { Adolescentes/jovens } \\
\text { americanos }(N=14276 ; \\
M=16.4 \text { e } M=21.9 \text { anos })\end{array}$ & DPS (indireto) \\
\hline & Kreager et al. (2016) & $\begin{array}{l}\text { Quantitativo - análise } \\
\text { questionários }\end{array}$ & $\begin{array}{l}\text { Adolescentes americanos } \\
(N=914 ; 11-16 \text { anos })\end{array}$ & DPS \\
\hline & Young et al. (2016) & $\begin{array}{l}\text { Quantitativo - PPT/ } \\
\text { /BSD e análise } \\
\text { questionários }\end{array}$ & $\begin{array}{l}\text { Adolescentes americanos } \\
(N=173 ; 12-17 \text { anos })\end{array}$ & $\begin{array}{l}\text { PSS conservador I } \\
\text { DPS (mulheres) }\end{array}$ \\
\hline & Lyons et al. (2011) & $\begin{array}{l}\text { Misto - análise itens } \\
\text { mensuráveis*, escalas } \\
\text { e entrevistas }\end{array}$ & $\begin{array}{l}\text { Mulheres adolescentes/jovens } \\
\text { americanas }(N=600 ; n=46 ; \\
12-19 \text { anos) }\end{array}$ & $\begin{array}{l}\text { PSS liberal I DPS } \\
\text { pessoal e social }\end{array}$ \\
\hline & $\begin{array}{l}\text { Gubrium e Torres } \\
\text { (2013) }\end{array}$ & $\begin{array}{l}\text { Qualitativo - análise } \\
\text { photovoice }\end{array}$ & $\begin{array}{l}\text { Adolescentes/jovens latino- } \\
\text {-americanos }(N=20)\end{array}$ & $\begin{array}{l}\text { DPS ligado a } \\
\text { cultura }\end{array}$ \\
\hline & Tong (2013) & $\begin{array}{l}\text { Quantitativo - análise } \\
\text { questionários }\end{array}$ & $\begin{array}{l}\text { Adolescentes/jovens } \\
\text { ascendência asiática (EUA) } \\
(N=1060 ; 11-18 \text { e 18-26 } \\
\text { anos) }\end{array}$ & $\begin{array}{l}\text { DPS não ligado a } \\
\text { cultura }\end{array}$ \\
\hline & $\begin{array}{l}\text { Chmielewski et al. } \\
\text { (2017) }\end{array}$ & $\begin{array}{l}\text { Qualitativo - análise } \\
\text { conteúdo noticioso }\end{array}$ & Artigos online EUA $(N=204)$ & DPS (indireto) \\
\hline & Ringrose et al. (2013) & $\begin{array}{l}\text { Qualitativo - análise } \\
\text { conteúdo grupos } \\
\text { focais e entrevistas }\end{array}$ & $\begin{array}{l}\text { Adolescentes ingleses } \\
(N=35 ; 12-15 \text { anos })\end{array}$ & DPS \\
\hline & $\begin{array}{l}\text { Mascheroni et al. } \\
\text { (2015) }\end{array}$ & $\begin{array}{l}\text { Qualitativo - análise } \\
\text { temática grupos focais } \\
\text { e entrevistas }\end{array}$ & $\begin{array}{l}\text { Adolescentes italianos, } \\
\text { espanhóis e ingleses ( } n=107 \\
n=50 ; 11-16 \text { anos) }\end{array}$ & $\begin{array}{l}\text { DPS I resistência } \\
\text { residual }\end{array}$ \\
\hline & Bongardt et al. (2017) & $\begin{array}{l}\text { Misto - análise } \\
\text { qualitativa e } \\
\text { quantitativa da } \\
\text { conversação }\end{array}$ & $\begin{array}{l}\text { Adolescentes/jovens } \\
\text { holandeses ( } N=61 \text { díades; } \\
16-21 \text { anos) }\end{array}$ & DPS (indireto) \\
\hline & Martin et al. (2014) & $\begin{array}{l}\text { Qualitativo - análise } \\
\text { de conteúdo noticioso }\end{array}$ & $\begin{array}{l}\text { Artigos imprensa escrita } \\
\text { (Inglaterra/Escócia) }(N=85)\end{array}$ & DPS (indireto) \\
\hline & Fjaer et al. (2015) & $\begin{array}{l}\text { Qualitativo - análise } \\
\text { de entrevistas }\end{array}$ & $\begin{array}{l}\text { Jovens noruegueses }(N=41 \\
18-19 \text { anos) }\end{array}$ & $\begin{array}{l}\text { PSS liberal I DPS } \\
\text { social }\end{array}$ \\
\hline & $\begin{array}{l}\text { Damme e Biltereyst } \\
\text { (2013) }\end{array}$ & $\begin{array}{l}\text { Qualitativo - análise } \\
\text { de grupos focais }\end{array}$ & $\begin{array}{l}\text { Adolescentes/jovens belgas } \\
(N=57 ; 14-19 \text { anos) }\end{array}$ & $\begin{array}{l}\text { PSS liberal I DPS } \\
\text { social I DPS } \\
\text { pessoal (residual) }\end{array}$ \\
\hline & Franz et al. (2016) & $\begin{array}{l}\text { Quantitativo - } \\
\text { metodologia crítica } \\
\text { quantitativa }\end{array}$ & $\begin{array}{l}\text { Jovens alemães e ingleses } \\
(N=65,16-19 \text { anos), }\end{array}$ & $\begin{array}{l}\text { PSS liberal } \\
\text { (indireto) }\end{array}$ \\
\hline
\end{tabular}




\begin{tabular}{|c|c|c|c|c|}
\hline & Autoria da pesquisa & Tipo de estudo/método & População/amostra & Resultados \\
\hline \multirow{9}{*}{ 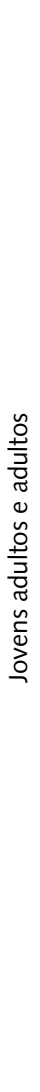 } & Sprecher et al. (2013) & $\begin{array}{l}\text { Quantitativo - itens } \\
\text { mensuráveis", escalas }\end{array}$ & $\begin{array}{l}\text { Estudantes universitários } \\
\text { americanos }(N=7777 \text {, } \\
18-22 \text { anos })\end{array}$ & DPS (indireto) \\
\hline & Boyer e Galupo (2015) & $\begin{array}{l}\text { Quantitativo - PPT/ } \\
\text { /BSD e análise de itens } \\
\text { mensuráveis* }^{\text {mens }}\end{array}$ & $\begin{array}{l}\text { Jovens adultos americanos } \\
(N=348 ; 18-25 \text { anos })\end{array}$ & DPS \\
\hline & Jones (2016) & $\begin{array}{l}\text { Quantitativo - PPT/ } \\
\text { MWSD e análise itens } \\
\text { mensuráveis* }^{*}\end{array}$ & $\begin{array}{l}\text { Jovens adultos e adultos } \\
\text { americanos ( } n=180 \\
M=31.9 \text { anos e } n=201 \\
M=33.5 \text { anos, para duas } \\
\text { condições experimentais) }\end{array}$ & DPS \\
\hline & $\begin{array}{l}\text { Esterline e } \\
\text { Muehlenhard (2016) }\end{array}$ & $\begin{array}{l}\text { Quantitativo } \\
\text { - questionários }\end{array}$ & $\begin{array}{l}\text { Estudantes universitários } \\
\text { americanos }(N=349, \\
17-35 \text { anos })\end{array}$ & DPS \\
\hline & $\begin{array}{l}\text { Montemurro e Siefken } \\
(2014)\end{array}$ & $\begin{array}{l}\text { Qualitativo - análise } \\
\text { de entrevistas }\end{array}$ & $\begin{array}{l}\text { Mulheres americanas } \\
(N=84,20-68 \text { anos })\end{array}$ & DPS \\
\hline & Lai e Hynie (2011) & $\begin{array}{l}\text { Quantitativo - PPT/ } \\
\text { /WSD e análise de } \\
\text { escalas }\end{array}$ & $\begin{array}{l}\text { Estudantes universitários } \\
\text { canadianos }(N=305, \\
17-25 \text { anos })\end{array}$ & DPS (indireto) \\
\hline & $\begin{array}{l}\text { Hackathorn e Harvey } \\
\text { (2011) }\end{array}$ & $\begin{array}{l}\text { Quantitativo - PPT/ } \\
\text { /BSD e análise de } \\
\text { escalas }\end{array}$ & $\begin{array}{l}\text { Estudantes universitários } \\
\text { americanos ( } N=115, \\
18-42 \text { anos, } M=20 \text { anos })\end{array}$ & $\begin{array}{l}\text { DPS (homens) } \\
\text { I DPS Inv. } \\
\text { (mulheres) }\end{array}$ \\
\hline & Papp et al. (2015) & $\begin{array}{l}\text { Quantitativo - PPT/ } \\
\text { /BSD e análise escalas/ } \\
\text { /questão aberta }\end{array}$ & $\begin{array}{l}\text { Estudantes universitários } \\
\text { americanos }(N=308 ; \\
18-25 \text { anos })\end{array}$ & $\begin{array}{l}\text { DPS Invertido } \\
\text { I DPS Social }\end{array}$ \\
\hline & Camoletto (2011) & $\begin{array}{l}\text { Misto - análise } \\
\text { inquéritos e entrevistas }\end{array}$ & $\begin{array}{l}\text { Jovens adultos e adultos } \\
\text { italianos ( } n=1000 ; 18-29 \\
\text { anos e } n=51 ; 18-34 \text { anos, } \\
\text { para cada método usado) }\end{array}$ & DPS (indireto) \\
\hline \multirow{4}{*}{ 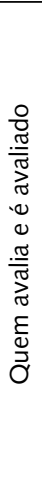 } & $\begin{array}{l}\text { Zaikman e Marks } \\
\text { (2016) }\end{array}$ & $\begin{array}{l}\text { Quantitativo - PPT/ } \\
\text { /BSD e análise de } \\
\text { escalas }\end{array}$ & $\begin{array}{l}\text { Jovens adultos americanos } \\
(N=596 ; M=29.2 \text { anos })\end{array}$ & $\begin{array}{l}\text { DPS I DPS } \\
\text { Invertido }\end{array}$ \\
\hline & Zaikman et al. (2016a) & $\begin{array}{l}\text { Quantitativo - PPT/ } \\
\text { /BSD e análise de } \\
\text { escalas }\end{array}$ & $\begin{array}{l}\text { Jovens adultos e adultos } \\
\text { americanos }(N=483 \\
M=33.3 \text { anos })\end{array}$ & $\begin{array}{l}\text { DPS I DPS } \\
\text { Invertido }\end{array}$ \\
\hline & $\begin{array}{l}\text { Sakaluk e Milhausen } \\
(2012)\end{array}$ & $\begin{array}{l}\text { Quantitativo - escalas } \\
\text { e tarefas de associação }\end{array}$ & $\begin{array}{l}\text { Estudantes universitários } \\
\text { canadianos }(N=103)\end{array}$ & $\begin{array}{l}\text { DPS I PSS liberal } \\
\text { (homens) I DPS } \\
\text { Inv. (mulheres) }\end{array}$ \\
\hline & Zaikman et al. (2016b) & $\begin{array}{l}\text { Quantitativo - PPT/ } \\
\text { /BSD e análise } \\
\text { questionários e itens } \\
\text { mensuráveis" }\end{array}$ & $\begin{array}{l}\text { Estudantes universitários } \\
\text { americanos }(N=154)\end{array}$ & $\begin{array}{l}\text { DPS I DPS } \\
\text { Invertido }\end{array}$ \\
\hline
\end{tabular}


cont.

\begin{tabular}{|c|c|c|c|c|}
\hline & Autoria da pesquisa & Tipo de estudo/método & População/amostra & Resultados \\
\hline \multirow{4}{*}{ 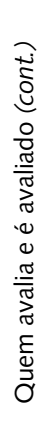 } & $\begin{array}{l}\text { Zaikman e Marks } \\
\text { (2014) }\end{array}$ & $\begin{array}{l}\text { Quantitativo - PPT/ } \\
\text { /BSD e análise } \\
\text { questionário/itens } \\
\text { mensuráveis" }\end{array}$ & $\begin{array}{l}\text { Estudantes universitários } \\
\text { americanos }(N=232)\end{array}$ & DPS (mais sexistas) \\
\hline & $\begin{array}{l}\text { Vrangalova et al. } \\
\text { (2014) }\end{array}$ & $\begin{array}{l}\text { Quantitativo - PPT/ } \\
\text { /BSD e escalas/ } \\
\text { questão aberta }\end{array}$ & $\begin{array}{l}\text { Estudantes universitários } \\
\text { americanos }(N=751)\end{array}$ & $\begin{array}{l}\text { PSS conservador } \\
\text { (menos } \\
\text { permissividade) }\end{array}$ \\
\hline & Ortiz et al. (2016) & $\begin{array}{l}\text { Quantitativo - análise } \\
\text { questionários }\end{array}$ & $\begin{array}{l}\text { Estudantes universitários } \\
\text { americanos }(N=313)\end{array}$ & $\begin{array}{l}\text { DPS (menos } \\
\text { permissividade) }\end{array}$ \\
\hline & Foster e Byers (2013) & $\begin{array}{l}\text { Quantitativo - PPT/ } \\
\text { /BSD e análise escalas } \\
\text { e questionários }\end{array}$ & $\begin{array}{l}\text { Estudantes universitários } \\
\text { canadianos }(N=207)\end{array}$ & $\begin{array}{l}\text { PSS conservador } \\
\text { (conservadores) }\end{array}$ \\
\hline
\end{tabular}

Fonte: Elaboração própria.

Nota: * Itens retirados de escalas/questionários/inquéritos ou desenvolvidos para os objetivos da investigação.

\subsubsection{Jovens adultos e adultos}

As provas de DPS são indiretamente inferidas por Sprecher et al. (2013), a partir das diferenças de género na permissividade sexual observada entre estudantes universitários americanos. Comparativamente com as mulheres, os homens mostraram maior aceitação da relação sexual ocasional, atitudes sexuais mais permissivas e uma sociosexualidade menos restritiva (e.g., comportamento sexual permissivo). Já outros trabalhos de investigação produzidos nos EUA reuniram prova mais concreta da manifestação do DPS entre jovens adultos e adultos, com ou sem relação com o género. Por exemplo, Boyer e Galupo (2015) observaram, entre outros aspetos, que o envolvimento sexual ocasional era avaliado como mais adequado para homens, do que para mulheres. Do mesmo modo, Jones (2016) concluiu sobre a presença do DPS na avaliação da desejabilidade de três alvos hipotéticos (PPT/Within Subject Design ou WSD) - com diferente número de parceiros e história de envolvimento em relações a três - para um potencial encontro com $/ \mathrm{sem}$ evolução para relação. Observou que as mulheres consideravam desejáveis alvos masculinos experientes, se atualmente monogâmicos, mas que alvos femininos experientes eram vistos pelos homens como menos desejáveis, mesmo quando monogâmicos "no presente". E ainda no mesmo sentido, Esterline e Muehlenhard (2016) apontaram para DPS, e particularmente DPS social, na avaliação do envolvimento em making out performativo (i.e., envolver-se íntima e publicamente com alguém com o objetivo de ser visto), que estudantes universitários perceberam ser uma atividade com consequências positivas/negativas para a reputação sexual de homens/mulheres 
e que tem, na sua base, motivações diferentes para homens (e.g., desejo de aprovação/estatuto) e mulheres (e.g., desejo de provocar ciúme, afirmar relacionamento ou estimular desejo masculino).

As conclusões sobre a emergência do DPS entre jovens adultos e adultos foram reforçadas em mais um estudo americano (Montemurro e Siefken, 2014) e um canadiano (Lai e Hynie, 2011), onde se enfatizou a relação com a variável idade. No primeiro, mulheres disseram perceber o termo cougar - mulheres maduras que procuram parceiros sexuais jovens - como negativo ou ter sentimentos mistos em relação ao mesmo; associaram-no a desvio e reconheceram que tanto podia ser uma forma de afirmar a sexualidade feminina como de reforçar o DPS (e.g., não há equivalente masculino). No segundo, com estudantes universitários, a evidência de DPS foi indiretamente mostrada por duas principais observações: 1) pessoas com idade superior a 65 anos e mulheres eram percebidas, respetivamente, como menos interessadas em sexo e como menos interessadas em práticas sexuais não convencionais; 2) homens jovens eram vistos como (os) mais interessados em sexo e práticas não convencionais e as mulheres como preferindo práticas convencionais.

Uma variação do DPS com padrões alternativos foi observada na avaliação de interações virtuais, em dois trabalhos de pesquisa conduzidos com estudantes universitários americanos. No estudo de Hackathorn e Harvey (2011), cenários hipotéticos de ciberinfidelidade (comportamento sexual mediado pelo computador ou sexo virtual) foram percebidos como infidelidade quando protagonizados por alvos do outro sexo (DPS homens/DPS Invertido mulheres) e considerados perturbadores e com potencial para levar à rotura do relacionamento. No estudo de Papp et al. (2015) observaram-se variações equivalentes, independentes do género, na avaliação de uma conversa Facebook fictícia (PPT) na qual homens ou mulheres (BSD) eram envergonhados/as ou humilhados/as - qualificados/as de sluts ou promíscuos/as - por outros homens ou mulheres (shamers ou críticos/as), com base no comportamento sexual descrito como desejado ou realizado. Avaliações negativas de homens sluts e de shamers de mulheres (DPS Invertido) foram acompanhadas por uma perceção dos shamers como mais críticos de mulheres do que de homens sluts, bem como de rotulagem diferencial de homens e mulheres como sluts (comportamentos "leves" das mulheres vs. "pesados" dos homens), indicadores de DPS social.

Por último, um estudo europeu (Camoletto, 2011) apontou indiretamente para DPS, tendo por base o tipo de guiões de interação sexual (script sexual) que jovens adultos indicaram ter adotado nas primeiras experiências sexuais. As referências a scripts sexuais genderizados (românticos/pragmáticos) 
foram dominantes e a maioria dos participantes associou scripts românticos (envolvimento emocional) a mulheres e scripts pragmáticos (desejo sexual/ /expetativa dos pares) a homens.

Em síntese, entre jovens adultos e adultos, especialmente americanos (sociedade mais representada nos estudos analisados), observou-se - tal como no grupo dos adolescentes e jovens - a emergência de DPS, mas a variação com padrões alternativos foi menos expressiva e limitada ao DPS Invertido (Tabela 1).

\subsubsection{DPS e as características de quem avalia e de quem é avaliado}

Um último conjunto de estudos mostrou não só a emergência/variação do DPS, como a influência das características de quem avalia e de quem é avaliado nesses processos. Sobre as características de quem é avaliado identificaram-se dois estudos com jovens adultos americanos, usando cenários hipotéticos e manipulação da informação sobre parceiros anteriores, aparência física e personalidade (Zaikman e Marks, 2016) e sobre violação/conformidade com género/heterossexualidade (Zaikman et al., 2016a). No primeiro, alvos femininos sexualmente muito ativos foram avaliados de forma mais positiva do que os equivalentes masculinos, quando a aparência e a personalidade eram ambas consideradas positivas ou negativas (DPS Invertido). No entanto, um DPS emergiu quando uma das características era positiva e a outra negativa, bem como quando se considerava um elevado número de parceiros sexuais. No segundo, a avaliação de mulheres "masculinas" e homens "femininos" mais experientes mostrou-se mais positiva que a de alvos do outro sexo que se conformavam ao género (DPS Invertido/DPS). E, no mesmo sentido, a avaliação de alvos masculinos homossexuais e femininos heterossexuais experientes revelou-se mais positiva que a de alvos femininos homossexuais (DPS) e masculinos heterossexuais (DPS Invertido), respetivamente.

Uma variação entre DPS e DPS Invertido (e PSS liberal) foi igualmente observada em dois estudos desenvolvidos com estudantes universitários canadianos (Sakaluk e Milhausen, 2012) e americanos (Zaikman et al., 2016b), cujo foco foram já as características de quem avalia. No primeiro caso, os investigadores observaram que, a um nível explícito, mulheres, mas especialmente homens, tendiam à aceitação do DPS enquanto, a um nível implícito, os homens tendiam à adoção de um PSS liberal e as mulheres de um DPS Invertido. Não confirmaram, contudo, a relação esperada entre desejabilidade social e padrões sexuais adotados. Já no segundo caso, os investigadores mostraram que a avaliação de alvos com mais/menos parceiros sexuais anteriores variava em função da qualidade da relação ou ligação afetiva (vinculação) estabelecida na infância com os progenitores, e na idade 
adulta com os parceiros românticos. Por um lado, observaram que, entre mulheres, mas não entre homens, uma vinculação romântica mais evitante (relutância a ser íntimo, confiar ou depender do parceiro) se associava a DPS, enquanto uma vinculação menos evitante se associava a DPS Invertido. Por outro, concluíram que, num subgrupo feminino, a avaliação de alvos experientes tendia a ser mais negativa para aqueles do mesmo sexo do progenitor com o qual a relação infantil havia sido pobre (DPS/DPS Invertido).

Ainda sobre as características de quem avalia, três estudos desenvolvidos com estudantes universitários americanos e um com estudantes canadianos destacaram o papel da permissividade nos padrões sexuais adotados e na sua tendência mais liberal ou conservadora. Nos trabalhos de Zaikman e Marks (2014) e Vrangalova et al. (2014), a avaliação de alvos hipotéticos com um número mais baixo ou elevado de parceiros sexuais apontou, respetivamente, para um DPS tradicional entre estudantes sexistas - avaliação de alvos femininos mais negativa que a de alvos masculinos experientes - e para um PSS conservador - avaliação de alvos sexualmente experientes mais negativa do que a de alvos menos experientes -, cujo pendor negativo se atenuava com graus mais altos de permissividade sexual, especialmente entre homens (indicador indireto DPS). Do mesmo modo, no trabalho de Ortiz et al. (2016) constatou-se que quanto mais positivas eram as atitudes dos estudantes face à pornografia (indicador de permissividade), menor era a probabilidade de aceitar o DPS. E ainda, no trabalho de Foster e Byers (2013), que explorou a associação entre atitudes sexuais, atribuição de culpa e perceção de estigmatização de alvos hipotéticos que contraíam uma infeção sexualmente transmissível que não o VIH (BSD/PPT), estigmatização e culpa mostraram-se superiores para alvos que se envolviam em sexo desprotegido do que para alvos cujo preservativo se rompia (PSS conservador), entre participantes conservadores.

Os últimos oito trabalhos de investigação sugeriram, pois, que as características de quem avalia e é avaliado podem influenciar a emergência do DPS, a variação com padrões alternativos - DPS Invertido - e a tendência liberal/ /conservadora do PSS, entre jovens adultos, especialmente entre estudantes universitários americanos e canadianos (Tabela 1).

\subsection{Emergências e variações particulares do DPS}

Nesta secção descreve-se a investigação que reúne evidência sobre a emergência/variação do DPS na avaliação do envolvimento de estudantes universitários - principalmente, mas não exclusivamente - em relacionamentos sexuais ocasionais, com alguns investigadores a distinguir entre relacionamentos ocasionais pontuais (bookups) e relacionamentos ocasionais que se repetem no tempo (Friends With Benefits ou FWB). 
Um primeiro conjunto de três trabalhos desenvolvidos com estudantes universitários americanos (e amostras da população geral) foi revelador da presença de DPS na avaliação da aceitação/rejeição de propostas para sexo ocasional (Conley et al., 2012), e na avaliação do potencial envolvimento de familiares/amigos nesse tipo de relacionamento (Rudman et al., 2012; Rudman e Fetterolf, 2014). Conley et al. (2012) mostraram, por um lado, que alvos femininos que aceitavam propostas hipotéticas para sexo ocasional eram avaliados de forma mais negativa do que alvos masculinos que o faziam. $\mathrm{E}$, por outro, que as participantes mulheres consideravam menos (e os homens mais) provável aceitar a proposta, com a antecipação de avaliações negativas pela aceitação a mediar parcialmente as diferenças de género observadas. Em amostras da população geral, os investigadores mostraram ainda que, ao rememorar propostas reais para sexo ocasional, as mulheres antecipavam uma avaliação mais negativa, do que os homens, pela aceitação, e que o medo da estigmatização mediava as diferenças de género na aceitação da proposta. Por seu lado, Rudman et al. (2012) e Rudman e Fetterolf (2014) concluíram que os homens tinham maior probabilidade de aceitar o DPS e de encorajar mais o envolvimento sexual ocasional de amigos/familiares homens, do que mulheres; sexismo hostil (antipatia face ao empowerment das mulheres) e DPS a mediar as diferenças de género. Por outro lado, indicadores indiretos de DPS foram visíveis nos argumentos considerados para dissuadir mulheres do envolvimento em relações ocasionais (Rudman et al., 2012). Prova de DPS social foi também reunida no trabalho de Farvid et al. (2017), mas neste, ao contrário dos anteriores, a variar com posições de resistência que, aparentemente, refletem um PSS mais liberal do que conservador. Mais especificamente, as narrativas de mulheres universitárias australianas sobre relacionamentos ocasionais refletiram resistência (e.g., DPS injustiça sexista) e acomodação ao DPS (e.g., preocupação com reputação sexual e silenciamento da ocasionalidade).

De entre a investigação conduzida com estudantes universitários americanos e que considerou, especificamente, os relacionamentos bookups, o trabalho de Hess et al. (2015) apontou de forma evidente para a presença de DPS nas estratégias usadas para dissuadir um/a amigo/a do envolvimento em hookups hipotéticos. As amigas eram alertadas para os riscos de se sentirem/serem vistas como sluts, de violação ou de contrair Infeções Sexualmente Transmissíveis (IST) e os amigos para o risco de a parceira ter IST, de acusação por violação ou rotulagem negativa pelo envolvimento com sluts. Um DPS Invertido residual foi ainda observado na rotulagem negativa dos homens (amigos/parceiros) envolvidos em bookups, mas a variação entre DPS e padrões alternativos foi mais evidente em outros 
estudos conduzidos com este grupo particular. Por exemplo, Reid et al. (2011) notaram a presença de um PSS tendencialmente liberal na avaliação do envolvimento em hookups, mas também consideraram estar presente um DPS tradicional na interpretação de um cenário hipotético descrevendo um encontro pós-bookup sem sexo (e.g., reparação da reputação sexual feminina vs. compaixão masculina). E, numa segunda exploração dos dados, Reid et al. (2015) sugeriram que, na base da avaliação diferencial observada, podia estar o código social que define como apropriado o sexo em bookups mas não em "primeiros encontros", especialmente entre mulheres (DPS). De forma semelhante, três estudos usando dados de um mesmo inquérito (Allison e Risman, 2013; England e Bearak, 2014; Kettrey, 2016) reuniram evidência de DPS a variar com um PSS conservador e um DPS Invertido. A resposta à pergunta sobre se respeitariam menos as mulheres/os homens pelo envolvimento em bookups com muitos parceiros apontou para a presença de um PSS, algo mais conservador que liberal, em metade ou mais das amostras. England e Bearak (2014) consideraram existir igual tendência na resposta à questão sobre o interesse em namorar pessoas com bookups frequentes (PSS conservador), identificando ainda um DPS (mais homens) e um DPS Invertido (mais mulheres). Já Kettrey (2016) e England e Bearak (2014) apontaram indiretamente para DPS tradicional na avaliação do envolvimento em hookups - um elevado número de mulheres afirmou que já se havia sentido menos respeitada por um parceiro bookup - mostrando que o mesmo comportamento pode associar-se a diferentes padrões sexuais - igualitários e diferenciais -, em função do tipo de indicador considerado. E essa variação é, adicional e indiretamente, revelada em dois últimos trabalhos, também conduzidos com estudantes universitários americanos. No trabalho de Wesche et al. (2016) observou-se que, quando consideradas de forma independente, as motivações sexuais pessoais e as atribuídas aos pares tinham maior probabilidade de ser género-concordantes (indicador indireto DPS) mas, quando comparadas, as primeiras pareciam mais femininas e as segundas mais masculinas, apontando uma possível crença na adesão dos pares à cultura bookup (indicador indireto de PSS liberal). No trabalho de Penhollow et al. (2017) observou-se, pelo contrário, um PSS mais conservador do que liberal na avaliação de cenários hipotéticos de envolvimento entre alvos femininos e masculinos, com manipulação da informação sobre relação, álcool e proteção - avaliação especialmente negativa de cenários que conjugavam hookups, uso de álcool e uso inconsistente do preservativo.

Por fim, estudos explorando os relacionamentos FWB, conduzidos com estudantes universitários canadianos - Weaver et al. (2011, 2013) - e com jovens adultos e adultos americanos - Lehmiller et al. (2011) -, 
apontaram mais para um PSS liberal do que para um DPS tradicional. Nas duas primeiras pesquisas, metade ou mais dos participantes considerou as experiências FWB positivas, acreditando, contudo que, o envolvimento das mulheres podia ter julgamento e efeitos/resultados mais negativos que o dos homens, e requerer maior secretismo (indicador indireto de DPS social). No último estudo, a evidência de DPS foi praticamente nula, destacando-se apenas algumas diferenças de género no que respeita a motivações, FWB simultâneos e expetativas relacionais.

Os trabalhos integrados nesta secção evidenciam a emergência de DPS na avaliação de relacionamentos ocasionais pontuais, como os hookups, e menos na de relacionamentos FWB. A variação com padrões sexuais alternativos foi igualmente observada, pese embora alguns deles se manifestassem apenas de forma residual ou indireta (Tabela 2).

TABELA 2 - Emergência e variação do DPS na avaliação da ocasionalidade, em contexto universitário particularmente

\begin{tabular}{|c|c|c|c|}
\hline $\begin{array}{l}\text { Autoria da } \\
\text { pesquisa }\end{array}$ & Tipo de estudo/método & População/amostra & Resultado \\
\hline $\begin{array}{l}\text { Conley et al. } \\
(2012)\end{array}$ & $\begin{array}{l}\text { Quantitativo - PPT/BSD, } \\
\text { memórias/experiências e } \\
\text { análise escalas }\end{array}$ & $\begin{array}{l}\text { Estudantes universitários } \\
(n=195 ; M=22 \text { anos, } \\
n=174 ; 18-54 M=24.8 \\
\text { anos) e população geral } \\
(n=2059 ; M=22.5 \\
\text { anos, } n=369 ; M=30 \\
\text { anos) americana }\end{array}$ & DPS \\
\hline $\begin{array}{l}\text { Rudman et al. } \\
\text { (2012) }\end{array}$ & $\begin{array}{l}\text { Quantitativo - análise } \\
\text { escalas/itens mensuráveis* }\end{array}$ & $\begin{array}{l}\text { Estudantes universitários } \\
\text { americanos }(N=503 ; \\
18-22 \text { anos })\end{array}$ & DPS (mais homens) \\
\hline $\begin{array}{l}\text { Rudman e Fetterolf } \\
\text { (2014) }\end{array}$ & $\begin{array}{l}\text { Quantitativo - análise } \\
\text { escalas/itens mensuráveis* }\end{array}$ & $\begin{array}{l}\text { Estudantes universitários } \\
\text { americanos }(N=225 \\
18-22 \text { anos }\end{array}$ & DPS (mais homens) \\
\hline Farvid et al. (2016) & $\begin{array}{l}\text { Qualitativo - análise } \\
\text { entrevistas }\end{array}$ & $\begin{array}{l}\text { Estudantes universitárias } \\
\text { australianas ( } N=15 ; \\
19-25 \text { anos) }\end{array}$ & $\begin{array}{l}\text { DPS social I PSS (liberal } \\
\text { indireto) }\end{array}$ \\
\hline Hess et al. (2015) & $\begin{array}{l}\text { Qualitativo - PPT/BSD e } \\
\text { análise questões abertas }\end{array}$ & $\begin{array}{l}\text { Estudantes universitários } \\
\text { americanos }(N=539 ; \\
M=19.50 \text { anos })\end{array}$ & $\begin{array}{l}\text { DPS I DPS Invertido } \\
\text { (residual) }\end{array}$ \\
\hline Reid et al. (2011) & $\begin{array}{l}\text { Qualitativo - PPT/BSD e } \\
\text { análise narrativas abertas }\end{array}$ & $\begin{array}{l}\text { Estudantes universitários } \\
\text { americanos }(N=273 ; \\
19 \text { anos ou menos) }\end{array}$ & PSS liberal I DPS \\
\hline Reid et al. (2015) & $\begin{array}{l}\text { Qualitativo - PPT/BSD e } \\
\text { análise narrativas abertas }\end{array}$ & $\begin{array}{l}\text { Estudantes universitários } \\
\text { americanos ( } N=273 ; \\
19 \text { anos ou menos) }\end{array}$ & DPS (indireto) \\
\hline
\end{tabular}


cont.

\begin{tabular}{|c|c|c|c|}
\hline $\begin{array}{l}\text { Autoria da } \\
\text { pesquisa }\end{array}$ & Tipo de estudo/método & População/amostra & Resultado \\
\hline $\begin{array}{l}\text { Allison e Risman } \\
\text { (2013) }\end{array}$ & $\begin{array}{l}\text { Quantitativo - análise } \\
\text { itens de inquérito }\end{array}$ & $\begin{array}{l}\text { Estudantes universitários } \\
\text { americanos }(N=24131 ; \\
18-21 \text { anos })\end{array}$ & $\begin{array}{l}\text { PSS (mais conservador } \\
\text { que liberal) }\end{array}$ \\
\hline $\begin{array}{l}\text { England e Bearak } \\
\text { (2014) }\end{array}$ & $\begin{array}{l}\text { Quantitativo - análise } \\
\text { itens de inquérito }\end{array}$ & $\begin{array}{l}\text { Estudantes universitários } \\
\text { americanos }(N=24289 \\
18-23 \text { anos })\end{array}$ & $\begin{array}{l}\text { PSS (mais conservador } \\
\text { que liberal) I DPS } \\
\text { (homens) I DPS Inv. } \\
\text { (mulheres) I DPS } \\
\text { (indireto) }\end{array}$ \\
\hline Kettrey (2016) & $\begin{array}{l}\text { Quantitativo - análise } \\
\text { itens de inquérito }\end{array}$ & $\begin{array}{l}\text { Estudantes universitários } \\
\text { americanos }(N=11077 ; \\
>25 \text { anos })\end{array}$ & $\begin{array}{l}\text { PSS (mais conservador } \\
\text { que liberal) I DPS } \\
\text { (indireto) }\end{array}$ \\
\hline $\begin{array}{l}\text { Wesche et al. } \\
\text { (2016) }\end{array}$ & $\begin{array}{l}\text { Qualitativo - análise } \\
\text { temática questões abertas }\end{array}$ & $\begin{array}{l}\text { Estudantes universitários } \\
\text { americanos }(N=154 ; \\
M=19.6 \text { anos })\end{array}$ & $\begin{array}{l}\text { DPS (indireto) I PSS } \\
\text { liberal (indireto) }\end{array}$ \\
\hline $\begin{array}{l}\text { Penhollow et al. } \\
\text { (2017) }\end{array}$ & $\begin{array}{l}\text { Quantitativo - PPT/BSD e } \\
\text { análise questionários }\end{array}$ & $\begin{array}{l}\text { Estudantes universitários } \\
\text { americanos }(N=574 ; \\
<25 \text { anos })\end{array}$ & $\begin{array}{l}\text { PSS (mais conservador } \\
\text { que liberal) }\end{array}$ \\
\hline $\begin{array}{l}\text { Weaver et al. } \\
(2011)\end{array}$ & $\begin{array}{l}\text { Misto - análise itens } \\
\text { mensuráveis* e entrevistas }\end{array}$ & $\begin{array}{l}\text { Estudantes universitários } \\
\text { canadianos }(N=26)\end{array}$ & $\begin{array}{l}\text { PSS liberal I DPS social } \\
\text { (indireto e residual) }\end{array}$ \\
\hline $\begin{array}{l}\text { Weaver et al. } \\
(2013)\end{array}$ & $\begin{array}{l}\text { Quantitativo - PPT/BSD e } \\
\text { análise itens mensuráveis" }\end{array}$ & $\begin{array}{l}\text { Estudantes universitários } \\
\text { canadianos }(N=404)\end{array}$ & $\begin{array}{l}\text { PSS liberal I DPS social } \\
\text { (indireto e residual) }\end{array}$ \\
\hline $\begin{array}{l}\text { Lehmiller et al. } \\
\text { (2011) }\end{array}$ & $\begin{array}{l}\text { Quantitativo - PPT/BSD e } \\
\text { análise itens mensuráveis" }\end{array}$ & $\begin{array}{l}\text { Jovens adultos e adultos } \\
\text { americanos }(N=411)\end{array}$ & $\begin{array}{l}\text { PSS liberal I DPS } \\
\text { (indireto e residual) }\end{array}$ \\
\hline
\end{tabular}

Fonte: Elaboração própria.

Nota: * Itens retirados de escalas/questionários/inquéritos ou desenvolvidos para os objetivos da investigação.

\section{Conclusão}

Esta revisão abrangente teve como objetivo compilar e sintetizar os resultados da investigação, produzida entre 2011 e 2017 em sociedades ocidentais, sobre a emergência do DPS e da sua variação com padrões sexuais alternativos.

De um modo geral, os trabalhos de pesquisa analisados mostram, à semelhança do observado em anteriores revisões da literatura (Bordini e Sperb, 2013; Crawford e Popp, 2003), que o DPS continua a ser um padrão fortemente enraizado em sociedades e culturas ocidentais, apesar da sua variação com padrões alternativos positivos - PSS liberal - e negativos PSS conservador e DPS Invertido. Tal como na investigação produzida antes de 2011, o DPS observado entre adolescentes e jovens, jovens adultos e adultos (e, neste caso, particularmente entre estudantes universitários), assume diferentes expressões - padrão pessoal vs. padrão social - e manifesta-se de forma mais direta, explícita, na aceitação, avaliação, julgamento e penalização diferencial do envolvimento de homens e mulheres em condutas sexuais 
ou sexualizadas diversas (e.g., relações sexuais na adolescência, número de parceiros e práticas sexuais, interação/comunicação em redes sociais/ /realidades virtuais e sexo ocasional). A emergência do DPS entre estudantes universitários, para o sexo ocasional pontual, vem ainda sublinhar um aspeto importante que diz respeito à aparente independência entre normalidade da conduta e normatividade da avaliação. Isto é, apesar de a ocasionalidade ser comum no contexto universitário (Garcia et al., 2012), ela não parece ter sido despenalizada, mantendo-se uma avaliação negativa, mais genderizada e/ou mais conservadora, do envolvimento nestes relacionamentos.

Comparada com as revisões da literatura antes referidas, esta revisão abrangente aponta para uma manifestação mais expressiva de DPS Invertido, e uma possível influência das características de quem avalia e é avaliado na emergência, variação e natureza mais liberal/conservadora dos padrões sexuais. Fatores como a idade ou o nível de compromisso são considerados por Crawford e Popp (2003), mas fatores como personalidade, aparência ou permissividade, aqui identificados, têm sido menos discutidos. Não obstante, o seu poder de influência revela, a par da diversidade de expressões e /manifestações, o caráter dinâmico dos padrões sexuais, da sua emergência/ variação, e a investigação futura deve considerar, por exemplo, o uso de métodos qualitativos para uma compreensão mais completa e integrada de DPS e padrões alternativos.

Por fim, a nossa revisão sugere a existência de especificidades sociais e culturais, indicando, por exemplo, a alternância de DPS com padrões positivos em sociedades não americanas, e com padrões negativos em sociedades americanas. Estas observações levam a supor que as sociedades americanas são marcadas por algum conservadorismo sexual que as distingue das restantes, mas esta é uma hipótese que carece de confirmação (e.g., emergência/ /variação do DPS em sociedades não americanas e estudos comparativos). O conservadorismo americano, pelo contrário, é, em parte, apoiado pela elevada evidência de DPS nos EUA, mas o argumento constitui, em si mesmo, uma limitação às nossas conclusões sobre emergência/variação do DPS em sociedades ocidentais. A estas juntam-se outras relacionadas com as opções metodológicas, como a exploração não exaustiva da qualidade da investigação incluída ou a exclusão de trabalhos escritos em outras línguas que não o inglês, e que podiam informar a questão da especificidade social e cultural, por exemplo.

Independentemente das limitações, a emergência observada de DPS e de padrões alternativos negativos mostra como, nas sociedades ocidentais, a experiência (hetero)sexual continua a ser marcada pela desigualdade de género e, muito provavelmente, por constrangimentos à liberdade, saúde e 
bem-estar sexual. Importa, por isso, agir na compreensão e na desconstrução destes padrões bem como na sensibilização para outros - igualitários e liberais - tendo sido objetivo desta revisão abrangente contribuir para a compreensão destes padrões sexuais.

Revisto por Ana Sofia Veloso

\section{Referências bibliográficas}

Allison, Rachel; Risman, Barbara (2013), “A Double Standard for "Hooking Up”: How Far Have We Come Toward Gender Equality?”, Social Science Research, 42(5), 1191-1206. Consultado a 13.05.2017, em https://www.sciencedirect.com/science/article/ pii/S0049089X13000665.

Arksey, Hilary; O’Malley, Lisa (2005), "Scoping Studies: Towards a Methodological Framework", International Journal of Social Research Methodology, 8(1), 19-32. Consultado a 19.01.2017, em https://www.tandfonline.com/doi/abs/10.1080/1364 557032000119616.

Blakemore, Judith E. Owen; Berenbaum, Sheri A.; Liben, Lynn S. (2008), Gender Development. New York: Taylor \& Francis.

Bongardt, Daphne van de; Reitz, Ellen; Overbeek, Geertjan; Boislard, Marie-Aude; Burk, Bill; Deković, Maja (2017), "Observed Normativity and Deviance in Friendship Dyads' Conversations about Sex and the Relations with Youths' Perceived Sexual Peer Norms", Archives of Sexual Behavior, 46, 1793-1806. Consultado a 13.05.2017, em https://link.springer.com/article/10.1007/s10508-016-0763-x.

Bordini, Gabriela Sagebin; Sperb, Tania Mara (2013), "Sexual Double Standard: A Review of the Literature Between 2001 and 2010", Sexuality \& Culture, 17, 686-704. Consultado a 02.09.2016, em https://link.springer.com/article/10.1007/ s12119-012-9163-0.

Boyer, C. Reyn; Galupo, M. Paz (2015), “Attitudes Toward Individuals in Same-Sex and Cross-Sex Encounters: Implications for Bisexuality”, Journal of Bisexuality, 15(1), 57-68. Consultado a 13.05.2017, em https://www.tandfonline.com/doi/abs/10.1080/ 15299716.2014.984371.

Camoletto, Raffaella Ferrero (2011), "Sexual Beginners: Accounting for First Intercourse in Italian Young People's Heterosexual Biographies”, Sex Education, 11(3), 315-325. Consultado a 13.05.2017, em https://www.tandfonline.com/doi/full/10.1080/14681 811.2011.590303.

Chmielewski, Jennifer; Tolman, Deborah; Kincaid, Hunter (2017), "Constructing Risk and Responsibility: A Gender, Race and Class Analysis of News Representations of Adolescent Sexuality", Feminist Media Studies, 17, 412-425. Consultado a 13.05.2017, em https://www.tandfonline.com/doi/abs/10.1080/14680777.2017.1283348. 
Conley, Terri D.; Ziegler, Ali; Moors, Amy C. (2012), "Backlash from the Bedroom: Stigma Mediates Gender Differences in Acceptance of Casual Sex Offers", Psychology of Women Quarterly, 37(3), 392-407. Consultado a 13.05.2017, em https:// journals.sagepub.com/doi/10.1177/0361684312467169.

Crawford, Mary; Popp, Danielle (2003), "Sexual Double Standards: A Review and Methodological Critique of Two Decades of Research", The Journal of Sex Research, 40(1), 13-26. Consultado a 28.07.2016, em https://www.tandfonline.com/doi/abs/ $10.1080 / 00224490309552163$ ?src=recsys.

Damme, Elke; Biltereyst, Daniel (2013), “Let's Talk about Sex: Audience Research of Flemish Teenage Television Viewers and Their View on Sexuality”, Journal of Youth Studies, 16(3), 287-303. Consultado a 13.05.2017, em https://www.tandfonline.com/ doi/full/10.1080/13676261.2012.710744.

England, Paula; Bearak, Jonathan (2014), "The Sexual Double Standard and Gender Differences in Attitudes Toward Casual Sex Among U.S. University Students", Demographic Research, 30(Art. 46), 1327-1338. Consultado a 13.05.2017, em https:// www.demographic-research.org/volumes/vol30/46/.

Esterline, Kate M.; Muehlenhard, Charlene L. (2016), "Wanting to Be Seen: Young People's Experience of Performative Making Out", The Journal of Sex Research, 54(8), 1051-1063. Consultado a 13.05.2017, em https://www.tandfonline.com/doi/abs/ 10.1080/00224499.2016.1242111.

Farvid, Panteá; Braun, Virginia; Rowney, Casey (2017), “'No Girl Wants to Be Called a Slut!': Women, Heterosexual Casual Sex and the Sexual Double Standard”, Journal of Gender Studies, 26(5), 544-560. Consultado a 13.05.2017, em https://www.tandfonline. $\mathrm{com} / \mathrm{doi} / \mathrm{full} / 10.1080 / 09589236.2016 .1150818$.

Fasula, Amy M.; Carry, Monique; Miller, Kim S. (2014), “A Multidimensional Framework for the Meaning of the Sexual Double Standard and Its Application for the Health of Young Black Women in the U.S", The Journal of Sex Research, 51(2), 170-183. Consultado a 13.05.2017, em https://www.tandfonline.com/doi/abs/10.1080/00224499.2012.716874.

Fjær, Eivind Grip; Pedersen, Willy; Sandberg, Sveinung (2015), “'I'm Not One of Those Girls': Boundary-Work and the Sexual Double Standard in a Liberal Hookup Context”, Gender \& Society, 29(6), 960-981. Consultado a 13.05.2017, em https:// journals.sagepub.com/doi/pdf/10.1177/0891243215602107.

Foster, Lyndsay R.; Byers, E. Sandra (2013), "Stigmatization of Individuals with Sexually Transmitted Infections: Effects of Illness and Observer Characteristics", Journal of Applied Social Psychology, 43(S1), E141-E152. Consultado a 13.05.2017, em https:// onlinelibrary.wiley.com/doi/full/10.1111/jasp.12036.

Franz, Anke; Worrell, Marcia; Vögele, Claus (2016), "Discourses of Sexual Relationships in a Sample of German and British Young People: A Q Methodological Study", Culture, Health \& Sexuality, 18(4), 391-404. Consultado a 13.05.2017, em https:// www.tandfonline.com/doi/full/10.1080/13691058.2015.1089324. 
Garcia, Justin R.; Reiber, Chris; Massey, Sean G.; Merriwether, Ann M. (2012), "Sexual Hookup Culture: A Review”, Review of General Psychology, 16(2), 161-176. Consultado a 04.12.2017, em https://journals.sagepub.com/doi/pdf/10.1037/a0027911.

Gubrium, Aline C.; Torres, M. Idali (2013), “The Message Is in the Bottle: Latino Youth Communicating Double Standard Ideologies through Photovoice", American Journal of Health Education, 44(3), 146-155. Consultado a 13.05.2017, em https://www. tandfonline.com/doi/full/10.1080/19325037.2013.767735.

Hackathorn, Jana; Harvey, Richard (2011), "Sexual Double Standards: Bias in Perceptions of Cyber-Infidelity”, Sexuality \& Culture, 15, 100-113. Consultado a 13.05.2017, em https://ink.springer.com/article/10.1007/s12119-010-9082-x.

Hess, Aaron; Menegatos, Lisa; Savage, Matthew (2015), "Shaming Jane: A Feminist Foucauldian Analysis of How College Students Employ the Sexual Double Standard in Peer Interventions", Women's Studies in Communication, 38(4), 462-485. Consultado a 13.05.2017, em https://www.tandfonline.com/doi/abs/10.1080/07491409.2015.1085476.

Howard, Judith A.; Hollander, Jocelyn A. (1997), Gendered Situations, Gendered Selves: A Gender Lens on Social Psychology. London: SAGE Publications.

Jones, Daniel (2016), “The 'Chasing Amy' Bias in Past Sexual Experiences: Men Can Change, Women Cannot”, Sexuality \& Culture, 20, 24-37. Consultado a 13.05.2017, em https://link.springer.com/article/10.1007\%2Fs12119-015-9307-0.

Kettrey, Heather Hensman (2016), "What's Gender Got to Do with It? Sexual Double Standard and Power in Heterosexual College Hookups", The Journal of Sex Research, 53(7), 754-765. Consultado a 13.05.2017, em https://www.tandfonline.com/doi/full/ 10.1080/00224499.2016.1145181.

Kreager, Derek; Staff, Jeremy; Gauthier, Robin; Lefkowitz, Eva; Feinberg, Mark (2016), "The Double Standard at Sexual Debut: Gender, Sexual Behaviour and Adolescent Peer Acceptance", Sex Roles, 75, 377-392. Consultado a 13.05.2017, em https://link. springer.com/article/10.1007\%2Fs11199-016-0618-x.

Lai, Yvonne; Hynie, Michaela (2011), "A Tale of Two Standards: An Examination of Young Adult's Endorsement of Gendered and Ageist Sexual Double Standards”, Sex Roles, 64, 360-371. Consultado a 13.05.2017, em https://link.springer.com/ article/10.1007\%2Fs11199-010-9896-x.

Lehmiller, Justin; VanderDrift, Laura; Kelly, Janice (2011), "Sex Differences Approaching Friends with Benefits Relationships", The Journal of Sex Research, 48(2-3), 275-284. Consultado a 13.05.2017, em https://www.tandfonline.com/doi/ full/10.1080/00224491003721694.

Lyons, Heidi; Giordano, Peggy; Manning, Wendy; Longmore, Monica (2011), "Identity, Peer Relationship and Adolescent Girl's Sexual Behavior: An Exploration of the Contemporary Double Standard", The Journal of Sex Research, 48(5), 437-449. Consultado a 13.05.2017, em https://www.tandfonline.com/doi/full/10.1080/0022 4499.2010.506679. 
Martin, Susan P.; McDaid, Lisa M.; Hilton, Shona (2014), "Double-Standards in Reporting of Risk and Responsibility for Sexual Health: A Qualitative Content Analysis of Negatively Toned UK Newsprint Articles”, BMC Public Health, 14, art. 792. Consultado a 13.05.2017, em https://bmcpublichealth.biomedcentral.com/ articles/10.1186/1471-2458-14-792.

Mascheroni, Giovanna; Vincent, Jane; Jimenez, Estefanía (2015), “'Girls Are Addicted to Likes so They Post Semi-Naked Selfies': Peer Mediation, Normativity and the Construction of Identity Online", Cyberpsychology: Journal of Psychosocial Research on Cyberspace, 9(1), art. 5. Consultado a 13.05.2017, em https://cyberpsychology.eu/ article/view/4329/3391.

McCarthy, Barry W.; Bodnar, L. Elizabeth (2005), "The Equity Model of Sexuality: Navigating and Negotiating the Similarities and Differences between Men and Women in Sexual Behaviour, Roles and Values", Sexual and Relationship Therapy, 20(2), 225-235. Consultado a 13.05.2017, em https://www.tandfonline.com/doi/ full/10.1080/14681990500113229.

McClintock, Elizabeth Aura (2011), "Handsome Wants as Handsome Does: Physical Attractiveness and Gender Differences in Revealed Sexual Preferences”, Biodemography and Social Biology, 57(2), 221-257. Consultado a 13.05.2017, em https://www.tandfonline.com/doi/full/10.1080/19485565.2011.615172.

Moher, David; Liberati, Alessandro; Tetzlaff, Jennifer; Altman, Douglas G.; The PRISMA Group (2009), "Preferred Reporting Items for Systematic Reviews and Meta-Analyses: The PRISMA Statement”, PLoS Medicine, 6(7), e1000097. Consultado a 13.05.2017, em https://doi.org/10.1371/journal.pmed.1000097.

Montemurro, Beth; Siefken, Jenna Marie (2014), "Cougars on the Prowl? New Perceptions of Older Women's Sexuality”, Journal of Aging Studies, 28, 35-43. Consultado a 13.05.2017, em https://www.sciencedirect.com/science/article/pii/ S0890406513000741.

Ortiz, Rebecca; White, Shawna; Rasmussen, Eric (2016), “Do Individual Perceptions Matter in Pornography Effects? How Perceived General Acceptance and Influence of Pornography May Impact Agreement with Sex-Roles Attitudes”, Communication Research Reports, 33(2), 88-95. Consultado a 13.05.2017, em https://www.tandfonline. com/doi/abs/10.1080/08824096.2015.1122583.

Papp, Leanna J.; Hagerman, Charlotte; Gnoleba, Michelle A.; Erchull, Mindy J.; Liss, Miriam; Miles-McLean, Haley; Robertson, Caitlin M. (2015), "Exploring Perceptions of Slut-Shaming on Facebook: Evidence for a Reverse Sexual Double Standard", Gender Issues, 32, 57-76. Consultado a 13.05.2017, em https://link.springer.com/ article/10.1007\%2Fs12147-014-9133-y.

Penhollow, Tina M.; Young, Michael; Nnaka, Tonychris (2017), “Alcohol Use, Hooking-Up, Condom Use: Is There a Sexual Double Standard?”, American Journal of Health Behavior, 41(1), 92-103. Consultado a 13.05.2017, em https://doi.org/10.5993/AJHB.41.1.10. 
Petersen, Jennifer L.; Hyde, Janet Shibley (2010), “A Meta-Analytic Review of Research on Gender Differences in Sexuality, 1993-2007”, Psychological Bulletin, 136(1), 21-38. Consultado a 13.05.2017, em https://pubmed.ncbi.nlm.nih.gov/20063924/.

Petersen, Jennifer L.; Hyde, Janet Shibley (2011), “Gender Differences in Sexual Attitudes and Behaviors: A Review of Meta-Analytic Results and Large Datasets", The Journal of Sex Research, 48(2-3), 149-165. Consultado a 13.05.2017, em https://pubmed.ncbi. nlm.nih.gov/21409712/.

Reid, Julie A.; Elliott, Sinikka; Webber, Gretchen R. (2011), "Casual Hookups to Formal Dates: Refining the Boundaries of the Sexual Double Standard”, Gender \& Society, 25(5), 545-568. Consultado a 13.05.2017, em https://journals.sagepub.com/doi/ pdf/10.1177/0891243211418642.

Reid, Julie A.; Webber, Gretchen R.; Elliott, Sinikka (2015), “'It’s Like Being in a Church and Being on a Field Trip:' The Date Versus Party Situation in College Students' Accounts of Hooking Up”, Symbolic Interaction, 38(2), 175-194. Consultado a 13.05.2017, em https://onlinelibrary.wiley.com/doi/full/10.1002/symb.153.

Reiss, Ira L. (1960), Premarital Sexual Standards in America. Glencoe, IL: The Free Press. Ringrose, Jessica; Harvey, Laura; Gill, Rosalind; Livingstone, Sonia (2013), "Teen Girls, Sexual Double Standards and 'Sexting': Gendered Value in Digital Image Exchange”, Feminist Theory, 14(3), 305-323. Consultado a 13.05.2017, em https://journals.sage pub.com/doi/pdf/10.1177/1464700113499853.

Rudman, Laurie A.; Fetterolf, Janell C. (2014), "Gender and Sexual Economics: Do Women View Sex as a Female Commodity?”, Psychological Science, 25(7), 1438-1447. Consultado a 13.05.2017, em https://journals.sagepub.com/doi/pdf/10.1177/0956797614533123.

Rudman, Laurie A.; Fetterolf, Janell C.; Sanchez, Diana T. (2012), "What Motivates Sexual Double Standard? More Support for Male Versus Female Control Theory”, Personality and Social Psychology Bulletin, 39(2), 250-263. Consultado a 13.05.2017, em https://journals.sagepub.com/doi/pdf/10.1177/0146167212472375.

Sakaluk, John K.; Milhausen, Robin R. (2012), "Factors Influencing University Students' Explicit and Implicit Sexual Double Standards”, The Journal of Sex Research, 49(5), 464-476. Consultado a 13.05.2017, em https://www.tandfonline.com/doi/abs/10.10 80/00224499.2011.569976.

Sprecher, Susan; Treger, Stanislav; Sakaluk, John K. (2013), "Premarital Sexual Standard and Sociosexuality: Gender, Ethnicity, and Cohort Differences", Archives of Sexual Behavior, 42, 1395-1405. Consultado a 13.05.2017, em https://link.springer.com/ article/10.1007\%2Fs10508-013-0145-6.

Tong, Yuying (2013), "Acculturation, Gender Disparity, and the Sexual Behavior of Asian American Youth”, Journal of Sex Research, 50(6), 560-573. Consultado a 13.05.2017, em https://www.tandfonline.com/doi/abs/10.1080/00224499.2012.668976.

Vrangalova, Zhana; Bukberg, Rachel E.; Rieger, Gerulf (2014), "Birds of a Feather? Not When It Comes to Sexual Permissiveness", Journal of Social and Personal 
Relationships, 31(1), 93-113. Consultado a 13.05.2017, em https://journals.sagepub. com/doi/10.1177/0265407513487638.

Weaver, Angela D.; Claybourn, Marvin; MacKeigan, Kelly L. (2013), "Evaluations of Friends-with-Benefits Relationship Scenarios: Is There Evidence of a Double Standard?", The Canadian Journal of Human Sexuality, 22(3), 152-159. Consultado a 13.05.2017, em https://utpjournals.press/doi/10.3138/cjhs.2128.

Weaver, Angela D.; MacKeigan Kelly L.; McDonald, Hugh A. (2011), "Experiences and Perceptions of Young Adults in Friends with Benefits Relationships: A Qualitative Study", The Canadian Journal of Human Sexuality, 20(1), 41-53. Consultado a 13.05.2017, em http://english2010information.pbworks.com/w/file/fetch/84700384/ Experiences \%20and\%20perceptions \%20of\%20young \%20adults \%20in \% 20 friedns \%20with\%20benefits \%20relationships.pdf.

Wesche, Rose; Espinosa-Hernández, Graciela; Lefkowitz, Eva S. (2016), “Gender's Role in Misperceptions of Peers' Sexual Motives”, Sexuality \& Culture, 20, 1003 -1019. Consultado a 13.05.2017, em https://link.springer.com/article/10.1007/ s12119-016-9370-1.

Young, Michael; Cardenas, Susan; Donnelly, Joseph; Kittleson, Mark J. (2016), "Perceptions of Peer Sexual Behavior: Do Adolescents Believe in a Sexual Double Standard?”, Journal of School Health, 86(12), 855-863. Consultado a 13.05.2017, em https://onlinelibrary.wiley.com/doi/full/10.1111/josh.12455.

Zaikman, Yuliana; Marks, Michael J. (2014), "Ambivalent Sexism and the Sexual Double Standard”, Sex Roles, 71, 333-344. Consultado a 13.05.2017, em https://link.springer. com/article/10.1007\%2Fs11199-014-0417-1.

Zaikman, Yuliana; Marks, Michael J. (2016), “The Influence of Physical Appearance and Personality on the Exhibition of the Sexual Double Standard", Sexuality \& Culture, 20, 255-276. Consultado a 13.05.2017, em https://ink.springer.com/article/10.1007/ s12119-015-9319-9.

Zaikman, Yuliana; Marks, Michael J.; Young, Tara M.; Zeiber, Jacqueline A. (2016a), "Gender Role Violations and the Sexual Double Standard”, Journal of Homosexuality, 63(12), 1608-1629. Consultado a 13.05.2017, em https://www.tandfonline.com/doi/ abs/10.1080/00918369.2016.1158007.

Zaikman, Yuliana; Vogel, Erin A.; Vicary, Amanda M.; Marks, Michael J. (2016b), “The Influence of Early Experiences and Adult Attachment on the Exhibition of the Sexual Double Standard", Sexuality \& Culture, 20, 425-445. Consultado a 13.05.2017, em https://link.springer.com/article/10.1007/s12119-015-9332-z. 
Artigo recebido a 04.09.2019

Aprovado para publicação a 20.01.2021

\author{
Helena Delgado Amaro \\ Faculdade de Psicologia e de Ciências da Educação, Universidade de Coimbra | Faculdade de \\ Psicologia, Universidade de Lisboa \\ Rua do Colégio Novo, 3000-115 Coimbra, Portugal \\ Contacto: helena.s.d.amaro@gmail.com \\ ORCID: https://orcid.org/0000-0002-0824-2827
}

\title{
Maria-João Alvarez
}

Faculdade de Psicologia, Universidade de Lisboa | Centro de Investigação em Ciência Psicológica CICPSI, Faculdade de Psicologia, Universidade de Lisboa

Alameda da Universidade, 1649-013 Lisboa, Portugal

Contacto: mjalvarez@psicologia.ulisboa.pt

ORCID: https://orcid.org/0000-0001-6048-3778

\section{Joaquim Armando Ferreira}

Faculdade de Psicologia e de Ciências da Educação, Universidade de Coimbra | Centro de Investigação em Neuropsicologia e Intervenção Cognitivo-Comportamental - CINEICC, Faculdade de Psicologia e de Ciências da Educação, Universidade de Coimbra

Rua do Colégio Novo, 3000-115 Coimbra, Portugal

Contacto: jferreira@fpce.uc.pt

ORCID: https://orcid.org/0000-0003-1733-5854

\section{Manifestation of the Sexual Double Standard in Western Societies (2011-2017): A Scoping Review}

This scoping review synthetizes research published between 2011 and $2017(n=46)$, that aimed to analyse emergencies and variations of the Sexual Double Standard (SDS) in western societies. A consistent emergence of the SDS is observed in the evaluation of different sexual contexts/ /behaviours. There is also evidence of positive (egalitarian, liberal) and negative (egalitarian and conservative, and reversed) alternative sexual standards. The emergence of the SDS and the variation with negative standards indicates that, in western societies, (hetero)sexual experience

\section{Manifestation du double standard sexuel dans les sociétés occidentales (2011-2017) : un examen de la portée}

Cet examen de la portée analyse les recherches publiées entre 2011 et 2017 ( $n=46)$ sur l'émergence et la variation du double standard sexuel (DSS) dans les sociétés occidentales. Une manifestation constante du DSS est observée dans l'évaluation de divers comportements sexuels. Il y a également des preuves de modèles sexuels alternatifs positifs (égalitaires libéraux) et négatifs (égalitaires conservateurs et inversés). L'émergence du DSS et la variation avec des modèles négatifs indiquent que, dans les sociétés occidentales, l'expérience (hétéro)sexuelle 
is marked by gender inequality and by restrictive and punitive sexual standards. Also, because these standards may limit sexual autonomy and men's and women's access to a positive sexuality, they must be deconstructed and replaced by others that are more equitable.

Keywords: gender; sexual behaviour; sexual double standard; sexuality. est marquée par l'inégalité de genre et par des modèles sexuels restrictifs et punitifs. Comme ces normes peuvent limiter la liberté sexuelle et l'accès des hommes et des femmes à une sexualité positive, elles doivent être la cible de déconstruction et de remplacement par d'autres, équitables. Mots-clés: conduite sexuelle; double standard sexuel; genre; sexualité. 